\title{
Exploration of Knowledge Acquisition Techniques in Tunnel Industry: The Case Study of Iran Tunnel Association
}

\author{
Mostafa Jafari (Associate professor) \\ Department of Industrial Engineering, Iran University of Science \& Technology \\ Iran, Tehran \\ Tel: 98-912-138-2658Ｅmail: Jafari@iust.ac.ir \\ Peyman Akhavan (Associate professor) \\ Department of Industrial Engineering, Iran University of Science \& Technology \\ Iran, Tehran \\ Tel: 98-912-336-0729 E-mail: akhavan@iuct.ac.ir \\ Maryam Akhtari \\ Department of Industrial Engineering, Iran University of Science \& Technology \\ Iran, Tehran \\ Tel: 98-912-337-1175 E-mail: akhtari.maryam@gmail.com
}

Received: January 4, 2011 Accepted: March 7, 2011 doi:10.5539/ijbm.v6n8p245

\begin{abstract}
Purpose: The main propose of this study is determining appropriate knowledge acquisition techniques to extract tunnel expert's knowledge.

Design/methodology/approach: A porposive sampling method was used and data were collected via face-to-face interview based on a validated Knowledge Acquisition Questionnaire (KAQ). A total of 33 experts in tunnel industry who were presented by Iranian Tunnel Associated were identified and selected.

Findings: The result of this study showed that semi-structured interview, timeline, think aloud problem-solving, commentary, teach back, concept map, process map, repertory grid technique, composition ladder, decision ladder, process ladder interview, matrix, Observational techniques have meaningful effects on elicitation of tunnel experts' knowledge.

Limitation: Briefly, problem of this project were large number of experts, Limited time for interviews, (In accordance to these experts' avocations and huge responsibility), and outspread geographical distribution (from Tehran, Khorasan, Khoozestan). Inadequate many experts know little about KM and it's advantages

Originality/value: The innovation of this research is the first time this kind of research has been done in Iran. Until now, in Iran any working has not been done in the field of management and extraction knowledge experts.

Keywords: Knowledge acquisition, Knowledge management, Knowledge elicitation

\section{Introduction}

In the 1970s, Artificial Intelligence (AI) practitioners came to believe that the secret to building effective software programs was to fill them with knowledge. However, they knew very little about the techniques and methods required to extract knowledge either from textural sources, databases or human experts. The field of knowledge acquisition and elicitation was born as a distinct area (Milton, 2007)

During the 1990s tools became available that significantly improve the inference and representation issues faced. The 1990s also saw the development of methods to formalise knowledge acquisition and the subsequent creation of a computer system. These methods of knowledge acquisition aim to improve: Maintainability, Visibility, Validation, and Cost (rols roise).

In all knowledge management projects there is a primary phase which is important in success of the project (Hashemian \& Afrazeh, 2006). For instance; Nick Milton 2007 believes" Knowledge acquisition includes the elicitation, collection, analysis, modeling and validation of knowledge for knowledge engineering and knowledge management projects." (Milton, 2007) Its objective is to reduce the communication gap between the expert or knowledge worker and the knowledge engineer, allowing the knowledge to become independent of its sources (Abela et al., 2005).
\end{abstract}


Knowledge Acquisition is famous for being known as an obstruction in the process of creating a Knowledge Base System. The main contributing factor in KA is the human aspect (Durkin, 1994).

Many knowledge systems contain knowledge-acquisition (KA) tools that help knowledge engineers and domain experts to build and maintain the system's knowledge base. They exploit a collection of acquisition techniques, which consist of a user interface, a prescribed procedure for using this interface, and a method for operational zing the acquired knowledge (Elppler, 2004).

On the other hand, Knowledge acquisition is used to elicit, transform, and transfer expertise from the knowledge source to the form of knowledge based systems (Durkin, 1994).

Different sources of knowledge used in KA: experts, documents, manuals, case studies etc (www.cs.man.ac.uk).

In this paper based on case study in Iran, the techniques of knowledge acquisition are briefly discussed and compared. This case study was conducted in accordance to efficiency in the field of knowledge acquisition (KA) in Iranian Tunneling Association. The main propose of this study is determine appropriate knowledge acquisition techniques to extract tunnel expert's knowledge. In this project the researchers found out that a combination of KA techniques in order to elicit both tacit and explicit knowledge of experts is needed.

The result of the previous study of implementing knowledge acquisition (KA) which is conducted in an Iranian petrochemical company showed that: a combination of KA techniques including semi structured interview, revised teach-back, commentary, laddering, repertory grid techniques in order to elicit both tacit and explicit knowledge of experts (Nezafati et al., 2007) .

\section{Literature review}

\subsection{Expert}

Expert is generally the primary source for the purpose of knowledge elicitation. The most common types of experts are: academics, practitioners, and samurai. Each of these types of expert differs along a number of dimensions. These include; the outcome of their expert deliberations, the problem solving environment they work in, the state of the knowledge they possess (both its internal structure and its external manifestation), their status and responsibilities, their source of information, the nature of their training. The academic type regards their domain as having a logically organized structure. Generalizations over the laws and behavior of the domain are important to them. Theoretical understanding is prized. Their knowledge is likely to be well structured and accessible. The practitioner classes are engaged in constant day-to-day problem solving in their domain. For them specific problems and events is the reality. The samurai is a pure performance expert - their only reality is the performance of action to secure an optimal performance. The knowledge elicitor must be alert to these differences because the various types of expert will perform very differently in KE situations (shadbolt \& Burton, 1995).

Knowledge typically could be acquired through one of two ways: either manual (through the knowledge engineer) or automatic. In the automated knowledge acquisition tools are developed to help the knowledge engineer or even the expert himself to build and maintain the required knowledge systems (Gruber, 1993b; Ahmed Rafea et al., 2003).

Now we should know Which KA technique to use: We need different techniques because there are different types of knowledge acquiring certain type knowledge is made more efficient using the right technique. Knowledge acquisition is commonly regarded as a major obstacle and bottleneck in the process of designing and implementing knowledge base. Failure to acquire and encode appropriate amounts of relevant knowledge lead to limited consultation performance of the system (Eriksson, 1991; Ahmed Rafea et al., 20031).

Most knowledge engineering projects involve $a$ knowledge elicitation or knowledge acquisition (KA) phase that often runs hand-in-hand with a knowledge modeling phase, the knowledge acquisition phase requires a significant degree of interaction between the knowledge engineer and the specialist. During this phase, the knowledge engineer uses techniques and tools to elicit tacit knowledge from discipline specialists. A diversity of knowledge acquisition tools is presented in the Knowledge Acquisition Matrix:

These tools manage the vast amount of complex and interrelated knowledge necessary to build a knowledgesystem and representing the knowledge in a form that can be used by a problem solver (Runkel \& Birmingham, 2002).

\subsection{Types of knowledge Acquisition techniques (Bechhofer)}

2.2.1 Protocol-generation techniques: The aim of these techniques is to produce a protocol, i.e. a record of behavior, whether in audio, video or electronic media. Audio recording is the usual method, which is then transcribed to produce a transcript (Epistemic). It is include various types of interviews (unstructured, semi-structured and structured), reporting techniques (such as self-report and shadowing) and observational techniques.

2.2.1.1 Interviews: The interview is the most commonly used knowledge elicitation technique and takes many forms, from the completely unstructured interview to the formally-planned, structured interview.KA technique 
in which the knowledge engineer asks questions of the expert or end user.

2.2.1.2 Unstructured Interview: An interview in which the knowledge engineer has no pre-defined questions. Basically a chat to find out broad aspects of the expert's knowledge.[PPT]expert and knowledge engineer are free to explore the domain. This type of interview can prove useful as an initial interview when known of the domain is little [Milton site].

2.2.1.3 Semi-structured interview: An interview in which pre-prepared questions are used to focus and scope what is covered Also involves unprepared supplementary questions for clarification and probing.[PPT] The questions for a semi-structured interview are ideally constructed some time before the interview and are sent to the expert so he/she can start to prepare responses.[Milton site]

2.2.1.4 Structured interview: An interview in which the knowledge engineer follows a pre-defined set of structured questions but can ask no supplementary questions (www.cs.man.ac.uk). A significant benefit of the structured interview is that it provides structured transcripts that are easier to analyze than unstructured conversation. (Hoffman et al., 1995; shadbolt, 1995) Often involves filling-in a matrix or generic headings. [PPT]

2.2.1.5 Observation: Simply observing and making notes as the expert performs their daily activities can be useful, although a time-consuming process. Videotaping their task performance can be useful especially if combined with retrospective reporting techniques (Epistemics).

2.2.1.6 Commentary: These techniques generate protocols by having the expert provide a running commentary on a typical task used in the domain (Epistemics).

In on-line PA the expert is being recorded solving a problem, and concurrently a commentary is made. The nature of this commentary specifies two sub-types of the on-line method (Shadbolt \& Burton, 1995). The basic technique here is the self-report:

2.2.1.7 Self report: The expert performing the task may be describing what they are doing as problem solving proceeds. This is called self-report (Shadbolt \& Burton, 1995). Experimental evidence has shown that self-reports can access cognitive processes that cannot be fully recalled without bias and distortion if explained after the task has been completed. A problem with the self-report technique is that of cognitive overload, i.e. the mental effort required by the expert to provide the commentary interrupts and affects their performance of the task. This is especially true in dynamic domains where time is critical. One way around this is to use an off-line reporting technique (Epistemics).

2.2.1.8 Shadowing: A variant on this is to have another expert provide a running commentary on what the expert performing the task is doing. This is called shadowing (Shadbolt \& Burton, 1995).

2.2.1.9 Off-line PA: allows the expert(s) to comment retrospectively on the problem solving session - usually by being shown an audio-visual record of it. This may take the form of retrospective self-report by the expert who actually solved the problem (Shadbolt \& Burton, 1995). An advantage of this is that the video can be paused or run at slow speed to allow time for full explanation. Variants of these reporting techniques involve a second expert commenting on another expert's performance (Epistemics) or there could be group discussion of the protocol by a number of experts including its originator (Shadbolt \& Burton, 1995).

2.2.2 Teach-back Technique: In the teach back technique, the knowledge engineer describes part of the knowledge that has been acquired during previous sessions or from other sources. The expert comments on what the knowledge engineer is describing to reveal misunderstandings (Epistemics) and clarifies terminology (www.cs.man.ac.uk). It involves asking patients to explain or demonstrate what they have been told (Barry \& Weiss, 2007).

2.2.3 Protocol analysis techniques: This type of technique is depending on the requirements of the project. For instance, more detailed categories will be used for the identification. Such categories may be taken from generic ontologies and problem-solving models (Epistemics). PA involves the identification of basic knowledge objects within a protocol, usually a transcript. For most projects, this makes use of categories of fundamental knowledge such as concepts, attributes, values, tasks and relationships (Epistemics). (PA) is a generic term for a number of different ways of performing some form of analysis of the expert(s) actually solving problems in the domain (Shadbolt \& Burton, 1995). These techniques such as goals, decisions, relationships and attributes. This acts as a bridge between the use of protocol-based techniques and knowledge modeling techniques (Epistemics).

2.2.4 Laddering techniques: Laddering techniques involve the construction, reviewing modification and validation of hierarchical knowledge, often in the form of ladders (i.e. tree diagrams).Here the expert and knowledge engineer both refer to a ladder presented on paper or a computer screen, and add, delete, rename or re-classify nodes as appropriate (Epistemics). Laddering means setting elements in a ladder according to a common criterion in order to visualize them (easier for the expert) and confirm model completion (and, in rule systems generate the knowledge in the form of rules).

2.2.4.1 Concept Ladder: shows classes of concepts and their sub-types. All relationships in the ladder, there is a relationship, also is more commonly known as a taxonomy and is vital to representing knowledge in almost all 
domains.

2.2.4.2 Composition ladder: shows the way a knowledge object is composed of its constituent parts. All relationships in the ladder are the part or part-of relationship. Also is a useful way of understanding complex entities such as machines, organisations and documents.

2.2.4.3 Decision Ladder: shows the alternative courses of action for a particular decision. It also shows the pros and cons for each course of action, and possibly the assumptions for each pro and con. It is a useful way of representing detailed process knowledge.

2.2.4.4 Attribute Ladder: shows attributes and values. All the adjectival values relevant to an attribute are shown as sub-nodes, but numerical values are not usually shown it is a useful way of representing knowledge of all the properties that can be associated with concepts in a domain.

2.2.4.5 Process Ladder: shows process (tasks, activities) and the sub-processes (sub-tasks, sub-activities) of which they are composed. All relationships are the part of relationship; it is a useful way of representing process knowledge.

2.2.5 Matrix-based techniques: It involves the construction of grids indicating such things as problems encountered against possible solutions. Important types include the use of frames for representing the properties of concepts and the repertory grid technique used to elicit, rate, analyze and categorize the properties of concepts.

2.2.5.1 Frames: Frames are a way of representing knowledge in which each concept in a domain is described by a group of attributes and values using a matrix representation. The left-hand column represents the attributes associated with the concept and the right-hand column represents the appropriate values. When the concept is a class, typical (default) values are entered in the right-hand column. The use of frames can also be adopted, although this would typically be used for validating previously acquired knowledge rather than for eliciting knowledge from scratch (Epistemics).

2.2.5.2 Timeline: A timeline is a type of tabular representation that shows time along the horizontal axis and such things as processes, tasks or project phases along the vertical axis. It is very useful for representing time-based process or role knowledge. It can also be used to acquire time-based knowledge. It is a simple representation that is often used in the early stages of knowledge elicitation to capture the basic of processes from the expert (Milton, 2007).

2.2.5.3 Matrix: A matrix is a type of tabular representation that comprises a 2-dimensional grid with filled-in grid cells. Ticks, crosses or comments in the matrix cells indicate which row object is applicable to which column object. Two kinds of matrix are attributed matrix and relationship matrix.

2.2.5.4 Forms: $A$ more recent form of knowledge model is the use of hypertext and web pages, in which relationships between concepts, or other types of knowledge, are represented by hyperlinks. This affords the use of structured text by making use of templates, i.e. generic headings. Different templates can be created for different knowledge types.

2.2.5.5 Repertory Grid technique: The Repertory Grid technique (Gaines, 1988; 1990), is based on the "Personal Construct Psychology" theory by Kelly (Kelly, 1955), which postulates that people view the world in terms of "constructs"(Montero et al)The technique is essentially matrix-based although it is more complex than simply filling-in a matrix of elements. Usually involves the following four main stages [Milton site] It encourages the classification of data using elements and constructs, such that the extent of mutual relationships and differences between entities or constructs are established (pages.cpsc.ucalgary.ca).

In this way, two constructs which are too similar (always the same ratings in different concepts/instances) may be identified as redundant, or may trigger the expert to find concepts that differentiate them.(Montero et al) The format of the repertory grid essentially guides the respondent in constructing his or her own questionnaire, while permitting comparisons across different people or groups.(pages.cpsc.ucalgary.ca).

2.2.6 Sorting techniques: It's used for capturing the way people compare and order concepts, and can lead to the revelation of knowledge about classes, properties and priorities (Bechhofer). Classification techniques aim to identify the terms and concepts of the domain and how these concepts are organized in classes, groups or components, according to the expert (Wright \& Ayton, 1987; Abel et al., 2005).

2.2.6.1 Card Sorting: Card Sorting techniques provide a means of achieving a more focused or systematic understanding of the classifications and relationships in the expert's domain (academic.cankaya.edu.tr).Here the expert is given a number of cards each displaying the name of a concept (Epistemics). The cards can name knowledge elements of any type such as objects, tasks, goals, actions, resources etc (Shadbolt \& Burton, 1995).

The expert has the task of repeatedly sorting the cards into piles such that the cards in each pile have something in common (Epistemics). This process is repeated many times. Using this task one attempts to get multiple views of the structural organization of knowledge by asking the expert to do the same task over and over again. Each time the expert sorts the cards he should create at least one pile that differs in some way from previous sorts. The expert should also provide a name or category label for each pile on each different sort (Shadbolt \& Burton, 
1995).

2.2.6.2 Triadic Elicitation Technique: This is a technique where the user is asked on what he thinks is similar and different about three randomly chosen concepts and in what way are two of them similar and different. This is also known as Three Card Trick, because three concepts are used and trick to elicit attributes that are not immediately and easily articulated by the expert (Epistemics). Experts are then asked to try and name the way in which the groups differ (academic.cankaya.edu.tr).

2.2.7 Limited-information and constrained-processing tasks: These techniques include the generation and use of network diagrams, such as concept maps, state transition networks and process maps (Milton, 2007). The use of these is particularly important in capturing the "what, how, when, who and why" of tasks and events. KA techniques that use knowledge models as the focus for discussion, validation and modification of knowledge (www.cs.man.ac.uk). the knowledge engineer elicits knowledge from the expert by mutual reference to a diagram on paper or computer screen, these techniques which either limit the time and/or information available to the expert when performing tasks that would normally require a lot of time and information to perform. This provides a quick and efficient way of establishing the key tasks and information used (Epistemics).

2.2.7.1 Twenty Questions: 20 questions (Grover, 1983), is an approach similar to the game of the same name. The expert is provided with little or no information about a particular problem to be solved. The expert must then ask the elicitor for specific information that will be required to solve the problem. The information that is requested, along with the order in which it is requested, provides the elicitor with an insight into the expert's problem solving strategy (Shadbolt \& Burton, 1995).key aspects, properties or categories and their relative priorities (Bechhofer). One difficulty with this method is that the elicitor needs a good understanding of the domain in order to make sense of the experts' questions, and to provide meaningful responses.

2.2.8 Diagram-based techniques: include the generation and use of concept maps, state transition networks, event diagrams and process maps. The use of these is particularly important in capturing the "what, how, when, who and why" of tasks and events.

2.2.8.1 Process Mapping: This type of diagram involves the construction, modification and validation of process maps, (www.cs.man.ac.uk) shows the inputs, outputs, resources, roles and decisions associated with each process or task in a domain and is an excellent way of representing information of how and when processes, tasks and activities are performed. The industry standard UML (Unified Modeling Language) makes use of process maps for functional modeling (Epistemics).

2.2.8.2 Concept Mapping: This involves the construction, modification and validation of concept maps(www.cs.man.ac.uk), shows knowledge objects as nodes and the relationships between them as links (usually labeled arrows). Any types of concepts and relationships can be used. it is very similar to a semantic network used in cognitive psychology. use of concept maps has been strongly advocated as a comprehensive technique for eliciting many types of knowledge. the industry standard UML (Unified Modeling Language) makes use of concept maps (combined with frames) for object knowledge(Epistemics).

2.2.8.3 State Diagram Mapping: that involves the construction, modification and validation of a state diagram. (www.cs.man.ac.uk) and comprises two elements: (1) nodes that represent the states that a concept can be in, and (2) arrows between the nodes showing all the events and processes/tasks that can cause transitions from one state to another. The industry standard UML (Unified Modeling Language) makes use of state transition networks for dynamic modeling (Epistemics).

\section{Methodology}

A cross sectional study was conducted among experts in tunnel industry, in Tehran city, from January 2009. A total of 33 experts in tunnel industry who were presented by Iranian Tunnel Association were identified and selected by using purposive sampling.

In general, this project was done in 5 steps:

1-in the first step, knowledge acquisition techniques were investigated and studied; and existing classifications were compared. At the end, we tried to use the classification which included more techniques as the base of the project and prepare a questionnaire including different techniques.

2-Secondly, a guideline about importance of knowledge in today's world, explaining the concepts of Knowledge Management, different types of knowledge and also different Knowledge acquisition techniques with examples were prepared.

3-In the third part, 33 experts in tunnel industry who were presented by Iranian Tunnel Committee were chosen to inter the survey. The condition for entrance to this survey was at least 10 design or executive projects. Most of the people mentioned above had 20 or more project in their resume.

4-The guideline was sent to be studied by the experts and finally by repeated calls, appointments for interviews were set.

5-In this step which was the most important and time consuming step of the project, all knowledge acquisition 
techniques were examined in accordance to efficiency in the field of knowledge acquisition for tunnel industry experts by doing individual structured interviews with all experts. Standardized 5-point Likert scales ranging from strongly effective to strongly ineffective ( 1 to 5 points) were used for each of the 22 items.

It is necessary to say that every session of interview lasted at least 2 hours and 72 hours of interviews were done for this project in general.

Before gathering the data, the validity of the questionnaire has tested by distributing it between 15 experts in both tunnel and managerial fields, also by factor analysis $(\mathrm{KMO}=0.659)$ and confirmed by Bartlett test: $(\chi 2=$ 1705.841, Sig $=0.000$ ) and the reliability has accepted by Cronbach's alpha $=0 / 8012>0.75$.

Data was analyzed using Statistical Package for Social Science (SPSS) program version 16. Data normality was tested using Kolmogorov-Smirnov procedure.

\section{Discussion}

Because of experts' time limitation, we need the techniques and tools which can include both Process and Concept knowledge; also these techniques and tools must be able to elicit both Explicit and Implicit knowledge. We need techniques which are able to present, analyze and categorize the tunnel knowledge in different phase:

Following the subjects described above, these techniques are selected:

semi structured interviews \& structured interviews Because: The main source of knowledge is human (experts), so we held it to communicate with them; think aloud problem-solving or commentary to capture the management knowledge and their ability in leadership to allow the expert to modify and expand on the knowledge already captured; teach-back techniques for reminding the knowledge presented by expert (or the others) in previous sessions; because the experts too busy; Composition Ladder to elicit the relationship between different knowledge of different experts and to determine the relation between different knowledge used in design; Decision Ladder Because it is a useful way of representing detailed process knowledge and shows the pros and cons for each course of action, and possibly the assumptions for each pro and con; Process Ladder Because it is a useful way of of representing process knowledge; Observation In activities which needed high technology, Videotaping expert s task performance can be useful especially if combined with retrospective reporting techniques; Timeline Because it is very useful for representing time-based process or role knowledge; Repertory grid technique since this technique has been selected to quantize the concepts and to elicit the tacit knowledge of experts and the way they categorize the concept in their mind; Process maps for functional modeling with this technique we can capture information of how and when processes, tasks and activities are performed; Concept maps for object knowledge, also to show concepts and relationships in tunnel industry; Observation In activities which needed to high technology, videotaping experts s task performance can be useful especially if combined with retrospective reporting techniques. We think that these techniques are applicable in similar industry with different branches in design engineering.

\section{Conclusion}

In accordance to meaningful levels which were obtained we are able to say:

With $99 \%$ of certainty semi-structured interview, timeline, think aloud problem-solving, commentary, teach back, concept map, process map, repertory grid technique, composition ladder, decision ladder \& process ladder techniques have meaningful effects on elicitation of tunnel experts' knowledge. And with $95 \%$ of certainty structured interview, matrix, Observational techniques have meaningful effects on elicitation of tunnel experts' knowledge. Also unstructured interview, Frames, Forms, card sorting, triadic elicitation, twenty-questions, concept ladder, attribute ladder techniques have no meaningful effects on elicitation of tunnel experts' knowledge.

It is necessary to add that the experts suggested that we use observation technique in the form of video recording only in projects with high scientific onus and high technology which was a descend beginning for explaining the technique.

Although triadic elicitation \& twenty-question techniques do not have any meaningful effect on knowledge elicitation, some experts suggested that we could use them in special cases. With obtained meaningful level of $95 \%$ (and even 99\%) of certainty we can say there is a meaningful difference between different technique sets which are used in elicitation of tunnel experts' knowledge; and in accordance to ranked averages obtained from Freedman test, these technique sets which are used in knowledge elicitation of tunnel industry experts has been ranked as below:

Protocol analysis techniques

Protocol-generation techniques

Diagram-based techniques

Matrix-based techniques

Sorting techniques 
Limited-information and constrained-processing tasks

\section{Limitation}

Briefly, problem of this project were large number of experts, Limited time for interviews, (In accordance to these experts' avocations and huge responsibility), and outspread geographical distribution (from Tehran, Khorasan, Khoozestan ...). Inadequate many experts know little about KM and its advantages.

\section{Further Investments and Studies}

Study on other eliciting techniques which we could not study because of time limit.

Study on automatic eliciting technique in another subject for further studies.

Pattern and methods of knowledge capture is a subject for investments in the field of knowledge elicitation and modeling according to them for elicitation of experts' knowledge in tunnel industry could be a title for a complement research.

Preparing parameters for measuring elicited knowledge is a brand new subject for a research.

Preparing standards to evaluated and judge the process of knowledge eliciting should be a complement research as a sequel for previous link.

Individual studies on knowledge eliciting methods in organizations which are leaders in the field of transforming and using knowledge management systems could be a good pattern for knowledge elicitation process design.

Study on other knowledge management tools in order to transforming incidental knowledge to clear knowledge which we could not study because of time limit.

Study on challenges which organizations will face in the process of transforming knowledge management and eliciting experts' knowledge.

Modeling of production of knowledge base organized culture.

Modeling of initiative processes in order to transform knowledge management in organization.

Beside these subjects, study on knowledge models including:

Modeling Language with Object Constraint Language, Common KADS Protégé 2000, Unified Multi-perspective Modeling, is suggested.

\section{Acknowledgements}

We thank all the experts who took part in the study and gratefully acknowledge management officers and staff of Faculty of industry, Iran University of Science and Technology.

\section{References}

Ahmed, R., Hesham, H., \& Hazmanc, M. (2003). Automatic knowledge acquisition tool for irrigation and fertilization expert systems. Expert Systems with Applications, 24, 49-57.

Barry, D., \& Weiss, M.D. (2007). Removing barriers to better, safer care, Health literacy and patient safety: Help patients understand, Manual for clinicians, American Medical Association Foundation and American Medical Association. Aspirus Wausau Hospital, Dr Joseph F Smith Medical Library Jan Kraus, MLS Library Manager \& Vol. Community Faculty UW Madison 2009 [Online] Available: http://aspiruslibrary.org/literacy/literacy.htm.

Bechhofer, Sean, Knowledge elicitation, COMP30411.

Durkin, J. (1994). Expert Systems. Design \& Development in Macmillan.

Elppler, M.J. (2004). Making Knowledge visible through Knowledge Maps: concepts, elements, cases. Edited by Holsapple, clyde, Hand Book on Knowledge Management. Springer-Verlag Berlin: Heidelberg, Pages 189.

Emberey, C.L., Milton, N.R., Berends, J.P.T.J., van Tooren, M.J.L., van der Elst, S.W.G., \& Vermeulen, B. (2007). Application of Knowledge Engineering Methodologies to Support Engineering Design Application Development in Aerospace. 7th AIAA Aviation Technology, Integration and Operations Conference (ATIO). Belfast, Northern Ireland.

Epistemics. (2002). Information on knowledge acquisition. [Online] Available: http://www.epistemics.co.uk/Notes/63-0-0.htm (November 2002).

Gaines, B. R. (1988). Knowledge acquisition systems for rapid prototyping of expert systems. INFOR, 26(4), pp256-285.

Gaines, B. R., and Linster, M. (1990). Development of second generation knowledge acquisition.

Grover, M. D. (1983). A pragmatic knowledge acquisition methodology. In Proceedings of the $8^{\text {th }}$ International Joint Conference on Artificial Intelligence, 436-438.

Gruber TR. (1993). A translation approach to portable ontology speciation's. Knowledge Acquisition, 5(2): $199\{220$.

Gruber, R. T. (1993b). Toward Principles for the Design for Ontologies used for Knowledge Sharing. The 
International Workshop on Formal Ontology, March 1993.

Hashemian Bojnord, N., \& Afrazeh A. (2006). Project Knowledge Management. International Journal WSEAS Transaction on Information Science and applications, pp 644-650.

Hoffman, R., Shadbolt, N.R., Burton, A.M., and Klein, G. (1995). Eliciting Knowledge from Experts: A Methodological Analysis. Organizational Behavior and Decision Processes, 62(2).

Janet E. Burge. Knowledge Elicitation Tool Classification, Artificial Intelligence Research Group, Worcester Polytechnic Institute.

Kelly, G. (1955). The Psychology of Personal Constructs. New York: Norton.

Luis Montero \& Colin T. Scott. Andersen Consulting, Improving the Quality of Component Business Systems with Knowledge Engineering, 161 North Clark St., IL 60601 USA, \{Luis.Montero, Colin.T.Scott\}@ac.com.

Mara Abela, T., Lu1's A. Lima Silvaa, b, John A. Campbellb, Luiz F. De Rosc. (2005). Knowledge acquisition and interpretation problem-solving methods for visual expertise: A study of petroleum-reservoir evaluation. Journal of Petroleum Science and Engineering, 47, 51-69.

Mehmet Nuri Cankaya. Knowledge Elicitation Techniques, These are the notes of (Dr.Mehmet Tolun and written by Mehmet Nuri Cankaya.), [Online] Available: http://pages.cpsc.ucalgary.ca/ saliu/repertory.htm.

Milton, N.R. (2007). Knowledge acquisition in practice: a step-by-step guide. Published by Springer, ISBN $1846288606,9781846288609$.

Nezafati N., Khadivar A., Afarideh E., \& Jalali S. M. J. (2007). A Method for Human Driven Knowledge Acquisition (Case Study in a Petrochemical Company), Information Technology Interfaces, 2008. ITI 2008. 30th International Conference.

Nielsen, J. (1997). Usability testing. In G. Salvendy (Ed.), Handbook of Human Factors and Ergonomics (2nd ed.). New York: John Wiley.

Ontology Engineering \& Ontology Acquisition. [Online] Available: www.cs.man.ac.uk/ carole/old/GGF/FinalTutorial/GGFpart4.ppt, pp.129-158. Academic Press 0749-5978/95.

Runkel, Jay T., \& Birmingham-William P. (2002). Building non-brittle knowledge-Acquisition tools, Electrical Engineering and Computer Science Department, University of Michigan, Ann Arbor, MI.

Shadbolt, N.R., and Burton, M. (1995). Knowledge elicitation: a systematic approach, in Evaluation of human work: A practical ergonomics methodology edited by J. R. Wilson and E. N. Corlett, systems. In B. Wielinga, J. Boose, B. Gaines, G, Schreiber and M van Someren (eds) Current Taylor and Francis, London, England, 1995. pp. 406-440. ISBN-07484-0084-2.

Trends in Knowledge Acquisition. IOS Press, Amsterdam.

Wright, G., \& Ayton, P. (1987). "The psychology of forecasting", in Wright, G., Ayton, P. (Eds), Judgemental Forecasting, Wiley, Chichester, pp. 83-105. 
Table 1. Knowledge Acquisition in a glance

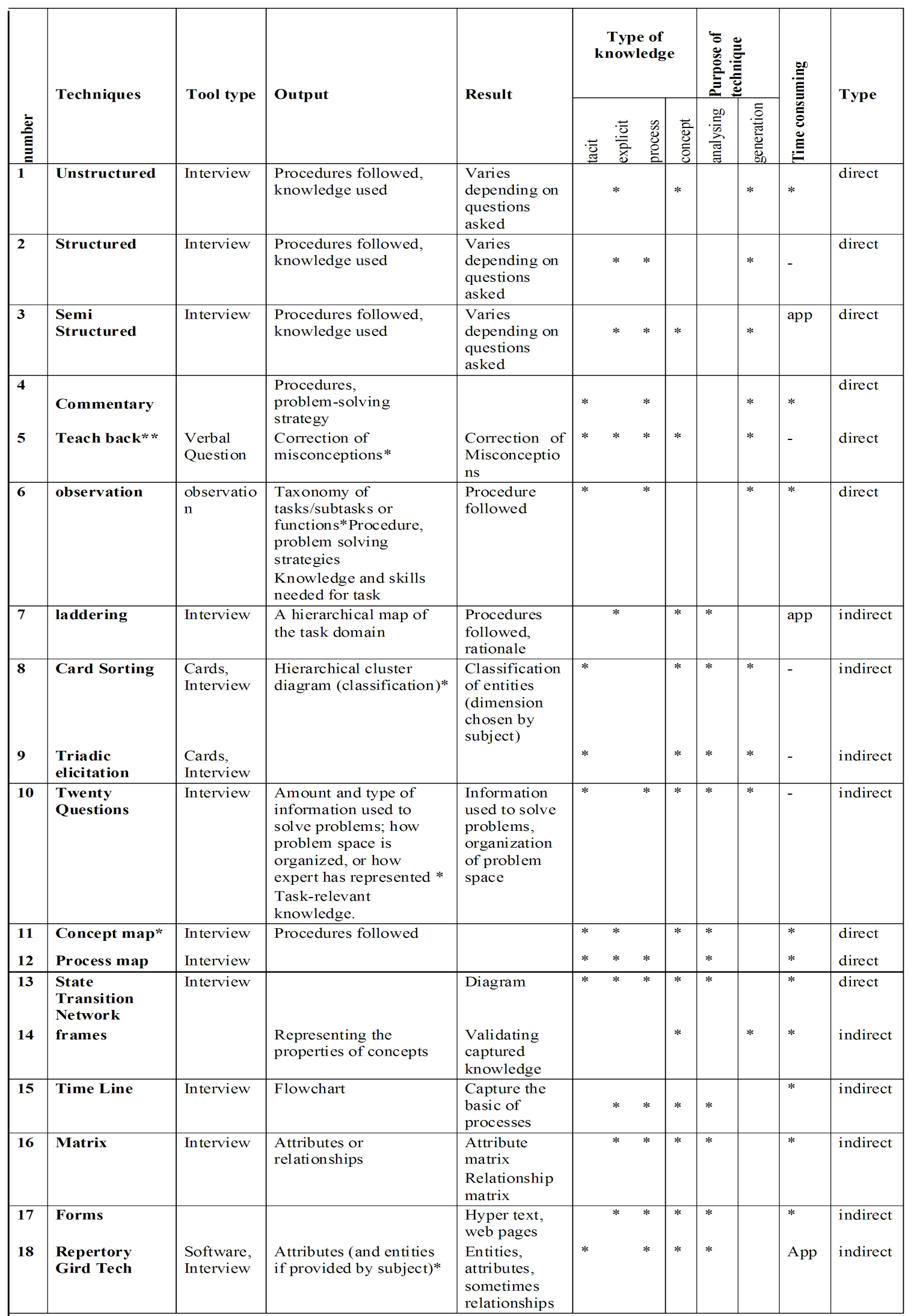




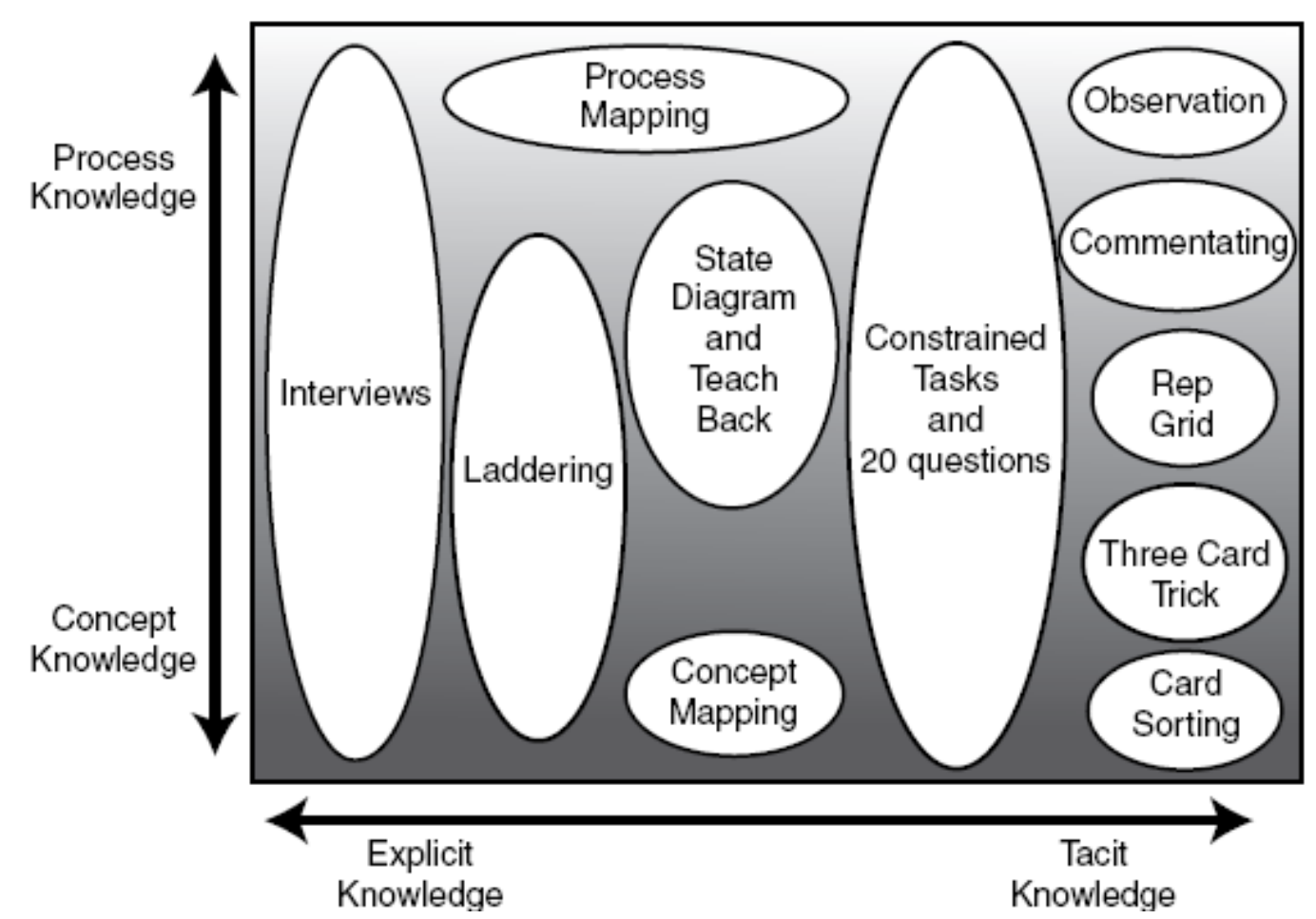

Figure 1. The Knowledge Acquisition Matrix provides several tools in order to acquire various types of knowledge. (Emberey et al., 2007) 